\title{
Central core disease of muscle with focal wasting
}

\author{
VICTOR DUBOWITZ AND MARGARET PLATTS
}

From the Department of Child Health and the Department of Medicine, University of Sheffield

In 1956 Shy and Magee described 'a new congenital non-progressive myopathy' in five members from three generations of the same family. Their ages ranged from 2 to 65 years. The pattern of inheritance suggested an autosomal dominant mechanism. The main clinical features were hypotonia and delayed motor milestones in infancy, and a mild nonprogressive weakness, affecting mainly proximal muscles, and the legs to a greater extent than the arms.

Histologically, a central area in the muscle fibre stained blue with Gomori's trichrome stain, in contrast to the red staining periphery of the fibre. This 'central core' consisted of compact myofibrils with cross striations. Some enlarged fibres contained more than one core.

A further case of central core disease was extensively investigated in England (Engel, Foster, Hughes, Huxley, and Mahler, 1961). This 21 -year-old man had clinical features similar to the cases of Shy and Magee (1965), but the family history was negative. Most fibres in the biceps brachii had a single central core, but in the vastus lateralis the majority of fibres had multiple cores. Histochemical studies on this "patient (Dubowitz and Pearse, 1960a) revealed that the central cores were devoid of enzyme activity, and that all the muscle fibres belonged to one enzyme type, in contrast to normal human muscle, in which at least two fibre types are recognizable with histochemical enzyme stains (Dubowitz and Pearse, 1960b). It was suggested that the cores were a nonfunctioning part of the muscle and this was supported by the electron microscopic observations of absence of mitochondria and sarcoplasmic reticulum in the core regions (Engel et al., 1961; Seitelberger, Wanko, and Gavin, 1961). Muscle biopsies from the parents of this patient were histologically and histochemically normal.

Bethlem and Meyjes (1960) reported a case from Holland. A 32-year-old woman had had a nonprogressive myopathy from birth. Biopsies of the triceps and quadriceps muscles showed a single central core in most fibres. The family history was negative.

We have recently diagnosed another case of central core disease. She presented in a rather unusual manner and the diagnosis was made, almost en passant, in the course of an abdominal operation. Histochemical investigations showed interesting differences from the case previously studied. The family history suggested an autosomal recessive pattern of inheritance.

\section{CASE HISTORY}

Kathleen W., a 40-year-old hospital cleaner, was admitted semi-comatose to a regional hospital on 27 December 1964. She could not give a coherent history, but had apparently been working until just before Christmas. She had then begun to vomit and became progressively more drowsy. She was found to be uraemic (blood urea $36 卬$ mg./100 ml., serum sodium 125 , potassium 5 , chloride 108 , and bicarbonate $5 \mathrm{mEq} . / 1$.). There was an associate $\$$ severe urinary infection. Despite treatment with intrad venous sodium bicarbonate and sodium chloride, which

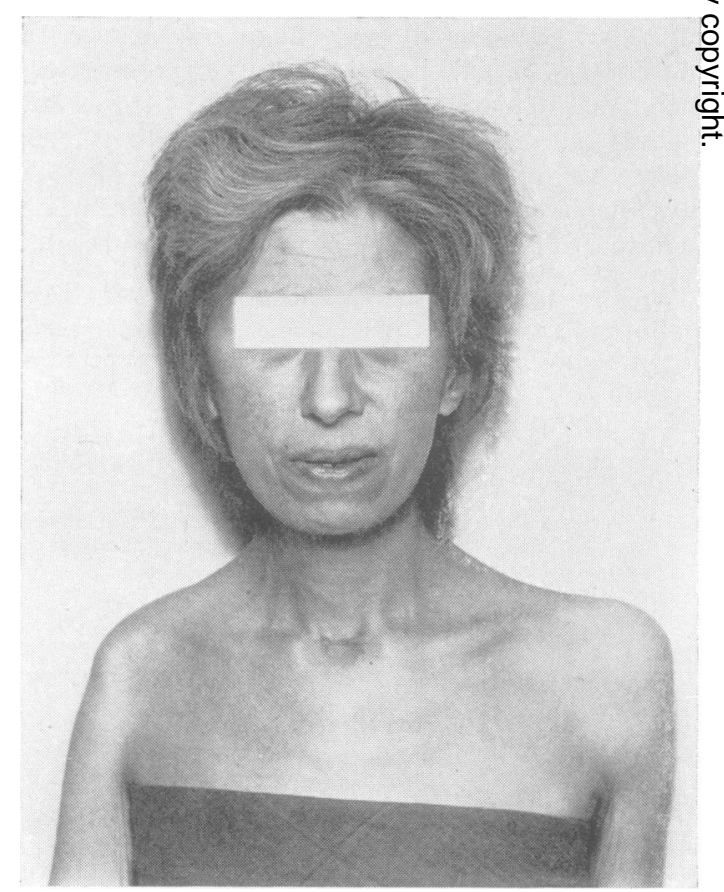

FIG. 1. Note the wasting of the right shoulder and the myopathic facies. 


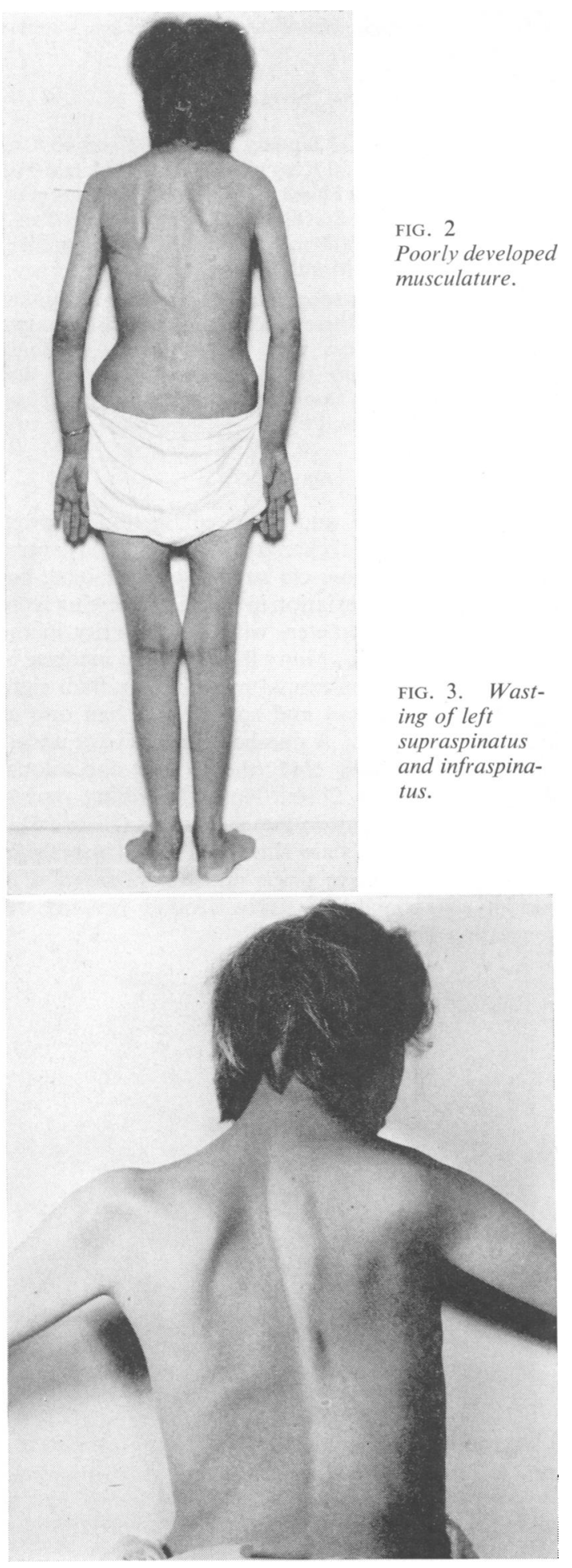

resulted in an improvement in the serum electrolytes, her clinical condition deteriorated and she was transferred to Sheffield on 30 December. At that stage she was in deep coma and moderately dehydrated. There was marked neck stiffness, but the limbs were hypotonic. The right arm was markedly wasted, particularly around the shoulder girdle (Fig. 1), and was 1 inch shorter than the left arm. The musculature generally was poorly developed (Figure 2).

Her urine was heavily infected. The blood urea level was $340 \mathrm{mg} . / 100 \mathrm{ml}$., the serum sodium 157 , potassium 3.4 , and bicarbonate $16.5 \mathrm{mEq} . / 1$. The cerebrospinal fluid contained 320 red blood cells and 2 white cells per c.mm., $70 \mathrm{mg}$. protein and $75 \mathrm{mg}$. sugar per $100 \mathrm{ml}$., and was sterile on culture.

A tracheostomy was performed the day after admission, because of accumulation of pharyngeal secretions. She had peritoneal dialysis on the second, third, and fourth days after admission. The urinary infection was treated with intramuscular colomycin.

By the fifth day, she was fully conscious. However, her limbs remained hypotonic and immobile. She had a myopathic facies, with diminished facial movement and inability to bury her eyelashes. The tendon reflexes were all very brisk; the plantar response was flexor. The cerebrospinal fluid was normal. Six days later she began to move her limbs and the power gradually improved. Her blood urea level had by this time fallen to $60 \mathrm{mg} . / 100 \mathrm{ml}$. and the serum electrolytes were normal, apart from a slightly low potassium level (3.9 mEq./1.). Her general condition continued to improve.

Further investigations revealed bilateral hydronephrosis and a ureteric obstruction on the right side. Four weeks after admission a ureteric stone was removed at laparotomy and biopsies were obtained from both rectus abdominis muscles.

Her muscle power continued to improve and she was walking with support a week after operation, and without support the following week, i.e., six weeks after the onset of the acute illness. At that stage she was still unable to get up from the floor and had great difficulty getting up from a chair. In addition to the wasting of the right shoulder there was focal wasting of the left supra- and infraspinatus (Fig. 3) and the glutei and lateral head of the hamstrings on both sides. Most muscle groups were grade 4 on the M.R.C. scale (Medical Research Council, 1942) but those of the right shoulder and also the extensors of the trunk and hips and the lateral hamstrings were only grade 2 to 3 . Slight facial weakness persisted. There was marked limitation of external rotation of the right shoulder, but other joints had a full range of movement. With the exception of the right triceps jerk, the tendon reflexes of the upper limbs were still brisk, but those of the lower limbs had become sluggish.

A detailed history from the patient, confirmed by her eldest sister, revealed that the wasting of the right shoulder girdle had been present since birth and had shown no change. The patient emphatically denied any previous weakness of muscles other than her right arm, and she had taken an active part in sports at school. She felt that she had not quite regained the strength she had before her illness. 
She was discharged on 13 February. When reassessed a month later, there was further improvement and she could go up and down steps. There was still some proximal weakness of the limbs. By mid-April she had shown further improvement, but was still having more difficulty in using the right arm than before the acute illness. The power in all muscle groups of the right arm was approximately grade 3 , with the proximal shoulder muscles weaker than the distal muscles. She was able to get up from the prone position, but leaned an elbow on her knee when doing so. She could ascend steps but still had slight difficulty. The facial muscles were normal. The tendon reflexes were depressed in the right arm but normal in the left arm and in the legs.

She was convinced that the residual weakness she still had had not been present before her recent illness. She is left-handed as well as left-footed and also uses the left eye for preference.

\section{FAMILY HISTORY}

A brother, Cyril W., aged 37 (Fig. 4, IV, 9), has also had a wasted right arm since birth. He is left-handed, but right-footed. He works as a furniture upholsterer and apart from the weakness of the right arm has never had any other weakness or disability.

On examination his pattern of wasting of the right shoulder was remarkably similar to his sister's. His right arm was 2 in. shorter than the left, $1 \frac{1}{2}$ in. being accounted for by the upper arm. In addition to the muscles of the right shoulder girdle, there was also wasting of the lower part of the right biceps, the right brachioradialis, and the left supra-and infraspinatus. These wasted muscles showed slight weakness, but all other muscle groups were normal. He got up from the prone position without any difficulty. Both his children were completely normal on examination.

The parents (Fig. 4, III, 1 and 2) were first cousins. According to the eldest sister, neither had any muscle weakness or wasting. The mother died suddenly of cerebral haemorrhage at 56, and the father at 81 (cause unknown).

No other members of the family had any muscle weakness or wasting. The two brothers who died (IV, 1

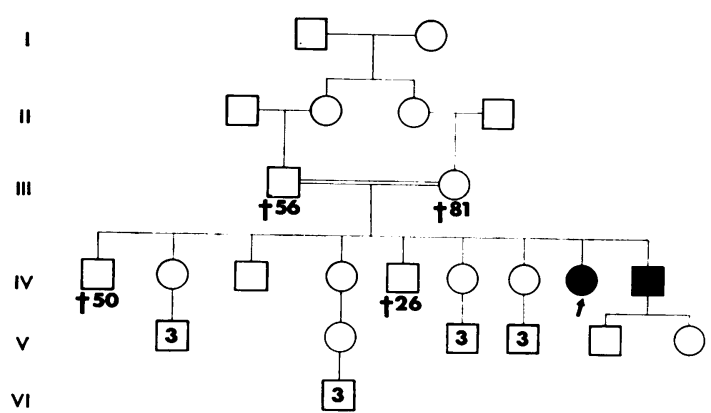

FIG. 4. Pedigree chart. and IV, 5) had carcinoma of the kidney and heart disease respectively.

\section{SPECIAL INVESTIGATIONS}

SERUM ENZYMES On 13 January 1965 SGOT was 40 King units $/ 100 \mathrm{ml}$.; SGPT $50 \mathrm{King}$ units $/ 100 \mathrm{ml}$; aldolase 11.0 units $/ \mathrm{ml}$./hr., creatine kinase $2.9 \mu \mathrm{M}$./ml./hr. (all normal), and one month later SGOT was $60 \mathrm{King}$ units $/ 100 \mathrm{ml}$.; SGPT 50 King units $/ 100 \mathrm{ml}$.; aldolase 12.5 units $/ \mathrm{ml} . / \mathrm{hr}$.; creatine kinase $1.0 \mu \mathrm{M} . / \mathrm{ml} . / \mathrm{hr}$.

MUSCLE BIOPSY The specimen of left rectus abdominis was subdivided into three blocks, and the right into two blocks. The specimens were rapidly frozen in liquid nitrogen and sections prepared for histological and histochemical studies along the lines previously described (Dubowitz and Pearse, 1964).

\section{HISTOLOGY}

With haematoxylin and eosin, all sections showed minimal pathological changes (Figure 5). The general architecture of the muscle bundles was retained, but there was marked variation in fibre size, ranging from 40 to $100 \mu$ in diameter, with the majority in the region of 40 to $60 \mu$. Many fibres had an increase in the number of sarcolemmal nuclei (more than eigh in transverse section) and some fibres had one o\& $\mathrm{O}$ more internal nuclei. A number of fibres were undere going phagocytosis and there were occasiona basophilic-staining fibres. Normal looking muscle 웡 spindles were present in two specimens (Figure 5).

The van Gieson stain showed a slight increase in perimysial connective tissue and a condensation of $\vec{\varnothing}$ endomysial connective tissue around isolated de- $\mathrm{G}$ generating fibres.

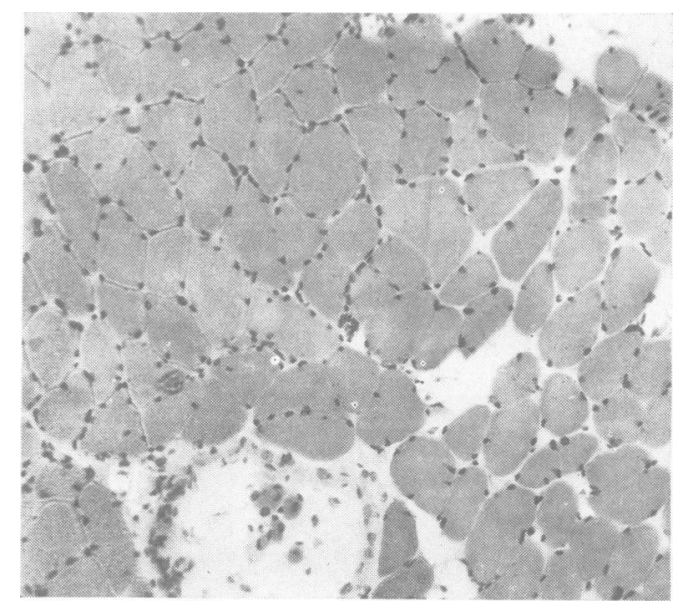

FIG. 5. Biopsy of left rectus abdominis, showing variation 0 ) in fibre size, increase in sarcolemmal nuclei, internal nuclei, normal muscle spindle. Haematoxylin and eosin $\times$ 100. 


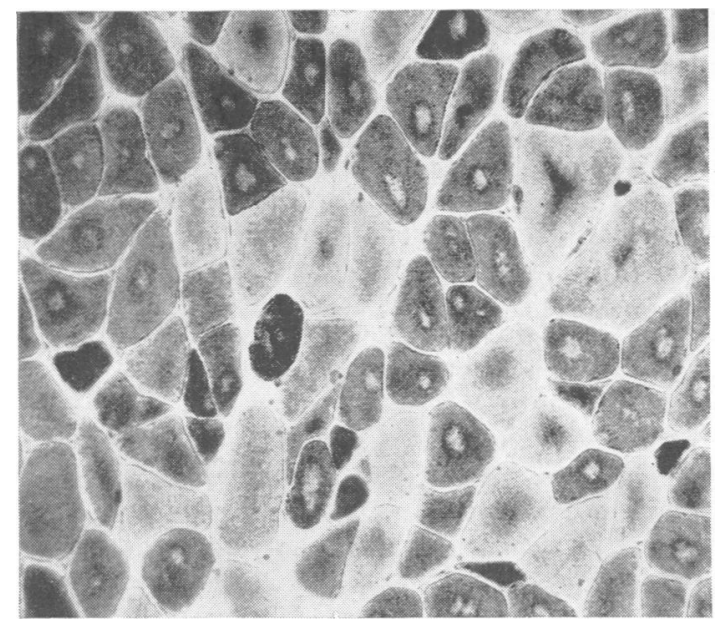

FIG. 6. Left rectus abdominis, showing the strongly and weakly reacting fibres. Central cores (devoid of stain) in darker staining fibres. Condensation of stain in centre of some of lighter fibres. NAD diaphorase (an oxidative enzyme $) \times 100$.

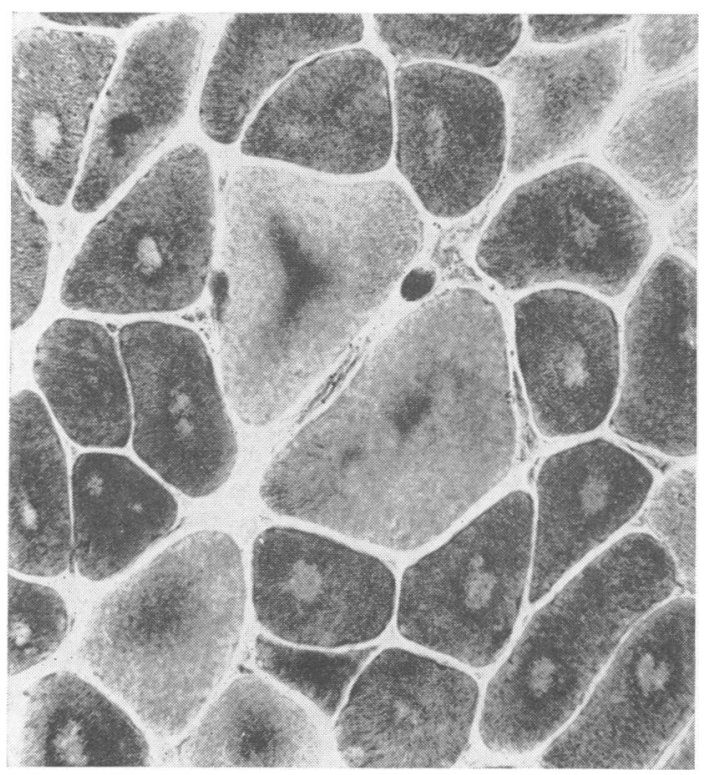

FIG. 7. Left rectus abdominis, showing central cores and central dark staining zones. NAD diaphorase $\times 200$.

\section{HISTOCHEMISTRY}

The reaction for nicotinamide-adenine dinucleotide (NAD) diaphorase, an oxidative enzyme, showed a striking picture (Figure 6). The muscle was divided into two fibre types, as in normal muscle, with a strong and a weak reaction respectively. Many fibres con- tained a single central core which was devoid of enzyme activity. These cores were practically confined to the fibres of the type reacting strongly for NAD diaphorase.

In longitudinal section the cores ran the length of the fibre. In the majority of fibres the cores were single, but occasional fibres had two cores. Most cores were $10-15 \mu$ in diameter, but some were up to $20 \mu$. They were usually about $10-15 \mu$ from the periphery of the fibre. One exceptional fibre, $100 \mu$ in diameter, had a $60 \mu$ core, but there were no others approaching this size. In the weaker reacting group of fibres, a number of fibres appeared to have a condensation of mitochondria in the central part of the fibre (Figures 6 and 7). This darker area also ran the length of the fibre in longitudinal section.

In one muscle spindle there was a suggestion of

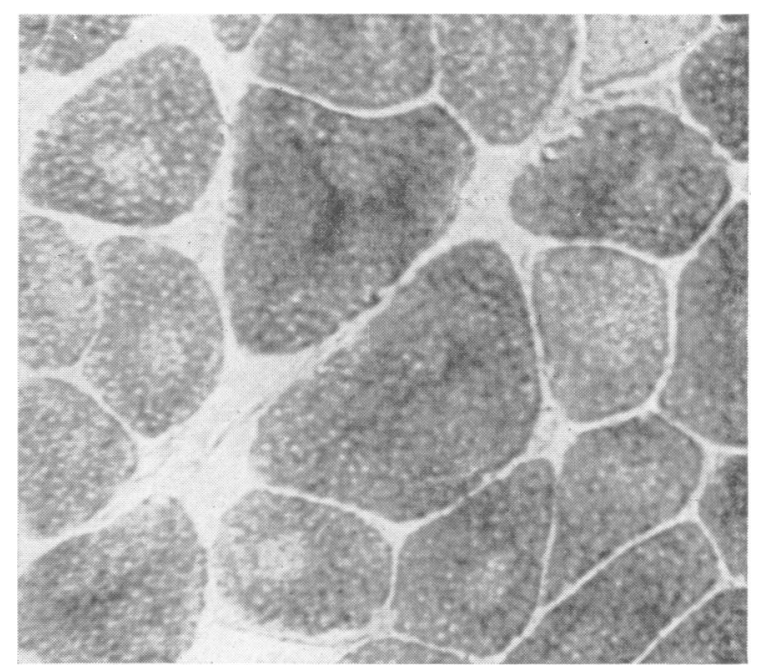

FIG. 8. Serial section to Fig. 7. Strongly and weakly reacting fibres. Same fibres show central cores or central dark staining zones as with NAD diaphorase. Phosphorylase $\times 200$.

central cores in three of the intrafusal fibres but this was not clear cut enough to be certain.

PHOSPHORYLASE As in normal muscle, there were strongly and weakly reacting fibres, and some intermediate in activity. Individual fibres usually had the reciprocal activity to the NAD diaphorase reaction. On serial section the same fibres as with the NAD diaphorase reaction had cores and these cores were devoid of phosphorylase activity (Figure 8). They showed up less strikingly than with the NAD diaphorase reaction, because it was the weakly reacting group which had the central cores. The 
dark central areas seen with the diaphorase reaction were also present in the same fibres with phosphorylase (Figure 8).

The cores also gave a negative reaction with the periodic-acid-Schiff reaction and with the reaction for myosin adenosine triphosphatase. With these reactions the cores were not as distinctly shown as with the NAD diaphorase.

Masson's trichrome stain confirmed the presence of the cores which stained red while the periphery of the fibre stained green.

\section{DISCUSSION}

Central-core disease cannot be recognized on clinical grounds alone, and the diagnosis rests on the demonstration of the cores by a trichrome stain or with histochemical enzyme stains. A number of aspects of the present case raise the question as to whether central core disease is in fact a uniform disease, or whether it is possibly a pathological manifestation of a number of syndromes.

The clinical appearance of this patient, with her poorly developed musculature, is very similar to the case of Engel et al. (1961). However, general wasting was not a feature of the original cases of Shy and Magee (1956) nor of the patient of Bethlem and Meyjes (1960).

The history of no previous weakness in our patient is unusual. All the previously described patients had some muscle weakness, and in the patient of Bethlem and Meyjes this appears to have been fairly severe.

None of the previously described cases had any focal wasting. The profound wasting of the right deltoid muscle in our case, as well as the focal wasting of other muscle groups, may be coincidental, but it seems reasonable to assume that they are connected with her generalized muscle disorder.

The gradual improvement in muscle power and tone as her renal function improved suggests that the profound weakness and hypotonia were precipitated by the uraemia. The relatively low potassium levels may have been an important factor, although the weakness persisted for a time after the potassium level was well within normal range. It is known that renal tubular acidosis may be associated with severe hypotonia (Dubowitz, 1963) or with weakness (Carroll and Davies, 1964), and the severe acidosis in this patient may also have been a contributing factor.

The distribution of the weakness was unlike that of a uraemic neuropathy and the very brisk tendon reflexes were also against this diagnosis. In previous cases of central core disease the tendon reflexes have been normal or sluggish. The brisk tendon reflexes in our patient appear to have been a temporary phenomenon in the acute phase.

Both the patient and her brother have a remarkably similar pattern of wasting of the various muscle groups, particularly of the right shoulder girdle. We do not know whether the brother has central core disease, as he was reluctant to have a biopsy. However, if one assumes that in the patient the focal wasting of muscle is part of her central core disease, it would then be reasonable to assume that the manifestations of the brother are also part of a central core disease.

The mode of inheritance of the disease in this family appears to be autosomal recessive. As both parents are dead one cannot with certainty exclude the presence of any muscle disease, but there is certainly no suggestive history. This makes a dominant inheritance unlikely. The pattern, therefore, differs from the family of Shy and Magee (1956). In the cases of Engel et al. (1961) and of Bethlem and Meyjes (1960), the family history was negative, which does not exclude an autosomal recessive mode of inheritance, but may alternatively represent a new mutation in a dominant inheritance.

The muscle biopsies showed evidence of minimap myopathic change on routine histological staining $\mathbb{R}$ and included a variation in fibre diameter, prolifera $\Omega$ tion of sarcolemmal nuclei with frequent internab nuclei, focal degeneration of muscle fibres, and sligh focal proliferation of endomysial connective tissue $\mathrm{O}$ These changes are similar to those noted in the previously described cases. The cores could not be seen at all in these sections, which illustrates how readily this disease can be missed in routine histological preparations.

Histochemical enzyme stains showed up the cores very clearly. As in the case previously studied (Dubowitz and Pearse, 1960a), the cores were completely devoid of enzyme activity. However, there was one striking difference between the two cases. The present patient showed a normal subdivision of the muscle fibres into at least two enzyme types, whereas in the previous patient all the fibres were of uniform enzyme activity and appeared to belong to one type only.

In the present patient the cores appeared to occur mainly in the fibres rich in NAD diaphorase and poor in phosphorylase. On serial sections it was shown that with different enzyme reactions it was the same fibres which had the central cores.

The majority of fibres had only a single core, occasional fibres had two cores, but none had more than two. This was similar to the biceps muscle of the previous case (Engel et al., 1961) but unlike the vastus lateralis of that patient, where the majority of fibres had multiple cores. 
The pathogenesis of the cores still remains obscure. Engel et al. (1961) discussed various hypotheses. In their patient, the uniformity of enzyme activity in all the muscle fibres suggests that there was an arrest in maturation before the time of normal differentiation of muscle, and recent work on enzymic histochemistry of human foetal muscle (Dubowitz, unpublished results) suggests that differentiation probably occurs after about 18 weeks' gestation. Thus, the presence of differentiation in the muscle of our present case suggests that the cores may have developed after differentiation into the fibre types had already occurred and to have then involved mainly one fibre type. Alternatively those fibres destined to form the type rich in NAD diaphorase and poor in phosphorylase may already have been selectively affected before the stage of differentiation into the two fibre types. Our recent studies on foetal muscle, as early as eight weeks' gestation, have shown that central cores are certainly not a feature of normal developing muscle at an early stage of its development.

\section{SUMMARY}

A 40-year-old woman was admitted in uraemic coma. She also had focal wasting of the right shoulder girdle from birth. After recovery from the uraemia, some generalized weakness persisted, and histochemical studies established a diagnosis of central core disease. A brother also has congenital focal atrophy of the shoulder girdle. The pattern of inheritance is probably autosomal recessive.

We wish to thank Miss J. Franks and Miss B. Warrington for technical assistance, Mr. J. Larway for the clinical photographs, Mr. A. T. Tunstill for the photomicrographs and $\mathrm{Mr}$. A. S. Foster for the pedigree chart. We are grateful for financial support from the Muscular Dystrophy Group of Great Britain and Sheffield University Research Fund.

\section{REFERENCES}

Bethlem, J., and Meyjes, F. E. P. (1960). Congenital, non-progressive central core disease of Shy and Magee. Psychiat. Neurol. Neurochir., (Amstr.), 63, 246-251.

Carroll, D., and Davies, P., (1964). Renal tubular acidosis presenting with muscle weakness. J. Neurol. Neurosurg. Psychiat., 27, 5-9.

Dubowitz, V. (1963). Problems of muscular hypotonia in infancy. In Research in Muscular Dystrophy; Proc. 2nd. Symposium, pp. 34-54, Pitman Medical Publ. Co., London.

lase in central-core disease of muscle. Lancet, 2, 23-24.

- - - (1960b). Reciprocal relationship of phosphorylase and oxidative enzymes in skeletal muscle. Nature (Lond.), 185, 701-702.

- - (1964). Histochemical aspects of muscle disease. In Disorders of Voluntary Muscle, pp. 194-219, edited by J. N. Walton. Churchill, London.

Engel, W. K., Foster, J. B., Hughes, B. P., Huxley, H. E., and Mahler, R.(1961). Central core disease-an investigation of a rare muscle cell abnormality. Brain, 84, 167-185.

Medical Research Council (1942). War memorandum No. 7. Aids to the Investigation of Peripheral Nerve Injuries. H.M.S.O., London.

Seitelberger, F., Wanko, T., and Gavin, M. A. (1961). The muscle fibre in central core disease. Histochemical and electron microscopic observations. Acta neuropath (Berl.), 1, 223-237.

Shy, G. M., and Magee, K. R. (1956). A new congenital non-progressive myopathy. Brain, 79, 610-621. 